\title{
Could the Use of Antihistamines Have Triggered Reversible Cerebral Vasoconstriction Syndrome? A Case Report
}

\author{
Sara Assadi ${ }^{a} \quad$ Latif Rahman $^{a} \quad$ Mark Kong $^{b} \quad$ Sukaina Asad ${ }^{c}$ \\ ${ }^{a}$ Acute Medicine Department, University Hospitals Leicester, Leicester, UK; ${ }^{b}$ Department of \\ Radiology, Oxford University Hospitals NHS Trust, Oxford, UK; ' ${ }^{2}$ epartment of Neurology, \\ University Hospitals of Leicester, Leicester, UK
}

\section{Keywords}

Reversible cerebral vasoconstriction syndrome $\cdot$ Headache $\cdot$ Diagnostic medicine $\cdot$

Anti-histamines

\begin{abstract}
A 38-year-old woman presented to the emergency department with recurrent severe headaches. Although initial computer tomography (CT) brain imaging was unremarkable, a later CT venogram demonstrated a small subarachnoid haemorrhage. Magnetic resonance angiogram (MRA) brain imaging subsequently confirmed reversible cerebral vasoconstriction syndrome (RCVS). In the acute setting, RCVS rarely falls into a differential diagnosis for headache presentations, as in this case. The radiological variability can make diagnosis of RCVS challenging. However, there are clinical consistencies that can aid physicians into accurately diagnosing RCVS. A thorough history, including a medication history, can help identify potential triggers of RCVS. As in this case, the combination of commonly used drugs, including antihistamines, provides a plausible trigger for RCVS. The direct vasoactive role of antihistamines is unclear, yet there is suggestion for its ability to potentiate the vasoactive action of other drugs. In this case we propose a causal relationship between the use of antihistamine and the development of RCVS.
\end{abstract}



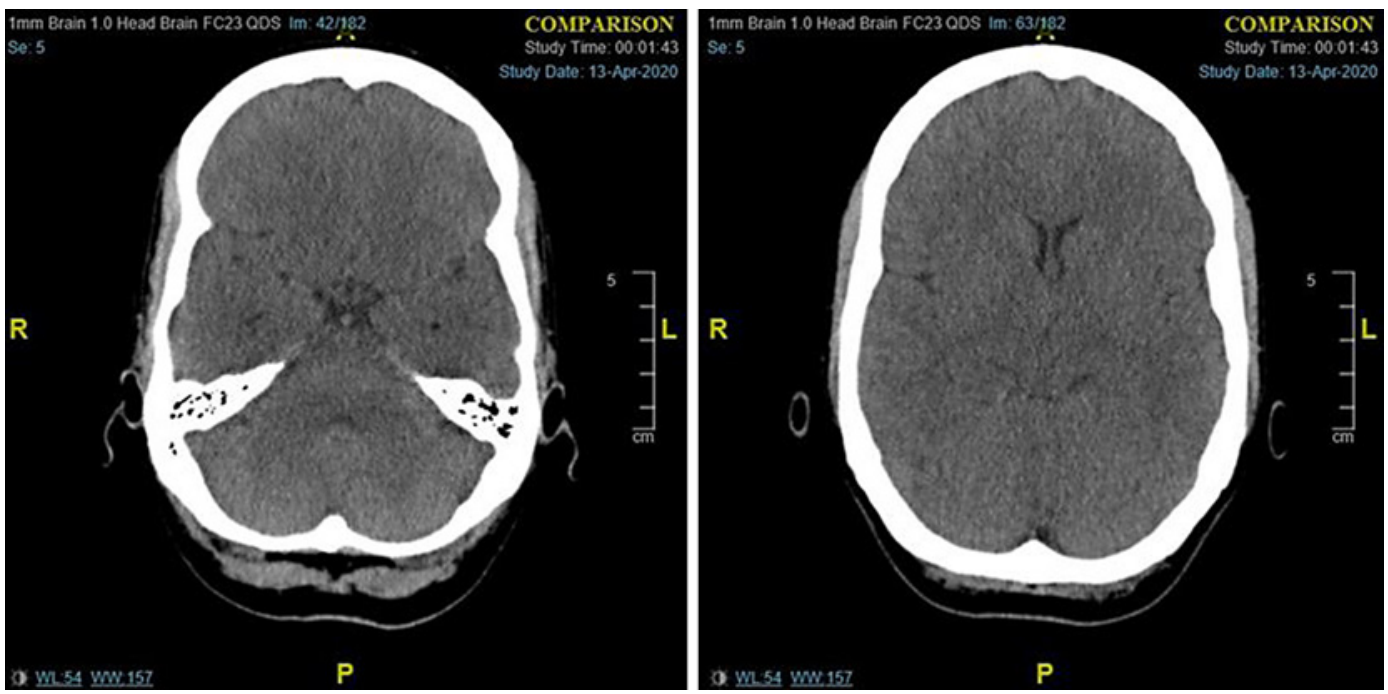

Fig. 1. CT head during first hospital admission. There was no major territory infarct, mass lesion, or intracranial haemorrhage demonstrated by the initial CT head.

\section{Introduction}

Reversible cerebral vasoconstriction syndrome (RCVS) is defined by the reversible vasoconstriction of cerebral arteries. In the majority of cases (90-95\%), individuals present with sudden severe headaches with features of thunderclap headache (TCH) that peak in intensity within 1 min [1]. Frequently, these headaches are described as recurrent in nature and are associated with an intermittent low grade, dull background headache that resolves spontaneously within weeks to a few months [1]. RCVS most commonly affects women aged 20-50 years [2]. Although idiopathic causes of RCVS can occur [3], up to $80 \%$ of cases involve well-recognized triggers of RCVS that include the use of vasoactive drugs, Valsalva manoeuvres (including coughing and sexual activity) and the postpartum period $[2,3]$.

\section{Case Presentation}

A previously healthy 38-year-old woman presented to the emergency department with a sudden onset, severe bi-frontal headache. This was aggravated by movement, light, and noise and associated with nausea and vomiting. The headache lasted a few hours and then resolved, leaving a mild residual band-like discomfort around her head. A CT head was performed within $4 \mathrm{~h}$ of the headache onset, reporting no major territory infarcts, intracranial haemorrhages, or mass lesions (Fig. 1).

Over the following two days, she developed two further headaches of similar presentation. Her examination and blood results were unremarkable, and she remained apyrexial throughout. After monitoring for a further few days, the medical team concluded the headaches were most likely migraines, and the patient was discharged.

After discharge, the patient experienced headaches on two further occasions, both resolving with simple analgesia. Four days later, the patient presented to hospital again with a further headache, this time persistent and more severe. Her vital signs and blood results were all normal. Overall, the clinical picture was almost identical to the initial 
Fig. 2. CT venogram on second hospital admission. The findings of the CT venogram were compared to the previous CT (Fig. 1). A new hyperdensity in the sulcal spaces of right parasagittal occipital region was noted, correlating with an acute subarachnoid haemorrhage in the right occipital lobe. There was no parenchymal low attenuation to suggest acute infarction. A venous sinus thrombus was also ruled out.
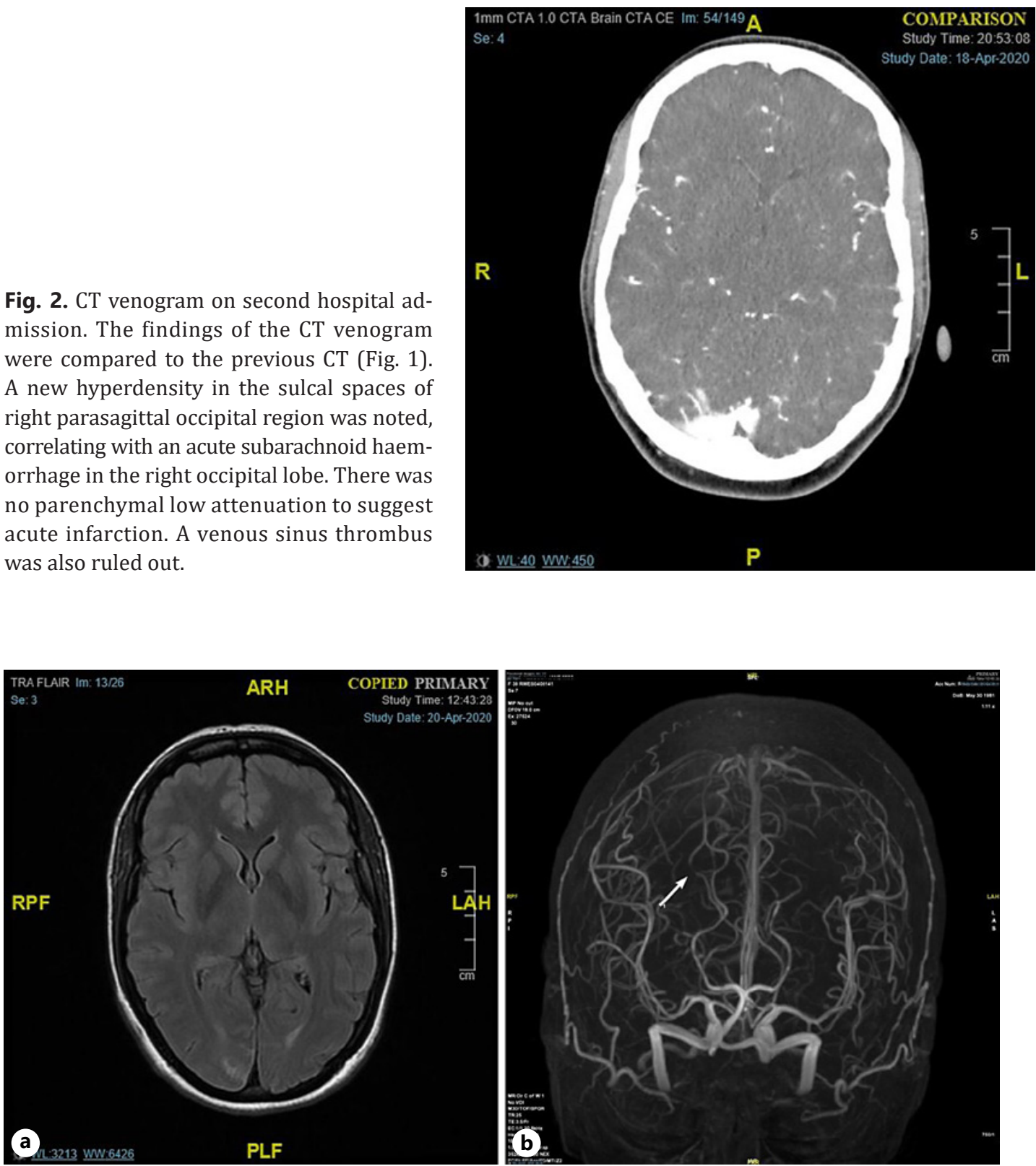

Fig. 3. MRI/MRA/MRV head with gadolinium. Flair imaging (image a) shows sulcal hyperintensity at the site of the limited right occipital SAH as demonstrated on the preceding CT head scan. The additionally obtained TOF-MRA sequence (image b) shows focal segmental narrowing in a distal branch of the right PCA (white arrow, image b). MRA, magnetic resonance angiogram; PCA, posterior cerebral artery.

presentation, with the exception of the headache being increasingly severe and prolonged in nature.

During the second admission, to rule out cerebral venous thrombosis, a CT venogram was performed and demonstrated a small subarachnoid haemorrhage in the right occipital lobe (Fig. 2). Subsequently, a magnetic resonance angiogram was performed which demonstrated focal segmental narrowing of the branch of the right posterior cerebral artery and thus confirmed RCVS (Fig. 3). 


\section{Discussion}

RCVS is a clinicoradiological syndrome characterised by recurrent, severe TCHs, with or without neurological symptoms, and multifocal cerebral arterial narrowing and dilatation. The prognosis of RCVS is excellent, with radiological and clinical features usually resolving spontaneously within 3 months $[1,3,4]$, as seen in this case. Unfortunately, RCVS can lead to a number of complications including cortical subarachnoid haemorrhages $(22 \%)$, intracerebral haemorrhages (6\%), seizures (3\%), and reversible posterior leukoencephalopathy (9\%) [1]. Almost half of patients have been found to develop neurological deficits as a result of RCVS [2]. One factor potentially contributing to this is a high frequency of misdiagnosis [2, 4].

Despite being well-known amongst neurologist, RCVS remains poorly understood and often misdiagnosed within the acute care setting. This may be influenced by the initial presentation clinically mimicking other common headache causes. Additionally, the radiological variability of RCVS and inconsistent duration between symptom onset and radiological findings can often make prompt diagnosis of RCVS challenging [5].

Misdiagnosis can come with consequences. The treatment for migraines often includes analgesic agents such as aspirin or triptans, both of which are contraindicated in RCVS. Triptans are vasoactive and have been found to exacerbate vasoconstriction and thus stroke risk [6], whilst aspirin can potentially precipitate complications of RCVS such as intracranial haemorrhage.

Although early diagnosis of RCVS may not be possible radiologically, awareness of the unique presentation and risk factors can aid clinicians. For example, RCVS most commonly affects women aged 20-50 years and presents with recurrent TCHs that intermittently resolve leaving a mild headache [2]. Most will suffer an average of 4 headaches over 1-4 weeks [2]. This case demonstrates all of these typical characteristics of RCVS.

A diagnostic tool was put in place by a recent retrospective study whereby a number of positive and negative predictors of RCVS were identified to propose an "RCVS2 score" (Table 1), which enables differentiation from other intracranial arteriopathies [7]. RCVS2 scores of $\geq 5$ are highly specific (99\%) and sensitive (90\%) for diagnosing RCVS, whereas scores $\leq 2$ are highly specific (100\%) and sensitive (85\%) for excluding RCVS [7]. In this case, the RCVS2 score was 5 (Table 1), making RCVS a strong differential diagnosis. Having this tool readily available to clinicians could improve diagnostics in future clinical practice and would have reduced the likelihood of misdiagnosis in this case.

Although idiopathic causes of RCVS can occur, up to $80 \%$ of cases involve well-recognised triggers $[3,4]$ (Table 2). In retrospect, this patient possessed a number of identifiable risk factors. Firstly, this patient had previously suffered from migraines. There is ample evidence supporting the link between migraines and RCVS, with one study finding that $40 \%$ of patients who developed RCVS, had previously suffered from migraines [2]. It is well-known that migraines are vascular headaches. During a migraine attack, cascades of events occur in which vasoactive neuropeptides are released and the trigeminovascular neurons are activated [8]. It is now thought that the pain caused by migraines is in part secondary to vasodilation of extracerebral vessels $[9,10]$. The vascular nature of migraines and the involvement of vasoactive neuropeptides, explains this association with RCVS. In addition, this patient was also on a selective serotonin-reuptake inhibitor, fluoxetine. RCVS has been found linked to serotonergic agents including fluoxetine in many cases $[3,4,6]$. Selective serotonin-reuptake inhibitors are well-known to have a vasoactive effect and thus carry the potential to drive cerebral vessels into vasospasm. Finally, after further detailed history taking, it became apparent that this patient had previously used marijuana, roughly 2 weeks prior to the headache onset. The link between marijuana and RCVS is well-known and again, this is secondary to the vasoactive potential of the drug [11].

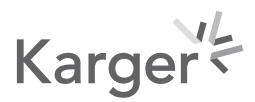


Table 1. RCVS2 score

\begin{tabular}{lcc}
\hline Criteria & Value & $\begin{array}{l}\text { Patient's } \\
\text { score }\end{array}$ \\
\hline Recurrent or single TCH & 5 & \\
Present & 0 & \\
Absent & & \\
Carotid artery (intracranial) & -2 & 0 \\
Affected & 0 & 3 \\
Not affected & 3 & \\
Vasoconstrictive trigger & 0 & 1 \\
Present & & \\
Absent & 1 & 1 \\
Sex & 0 & 0 \\
Female & & \\
Male & 1 & \\
Subarachnoid haemorrhage & 0 & \\
Present & & \\
Absent & & \\
\hline $\begin{array}{l}\text { Adapted from Ref. [7]. } \\
\text { RCVS, reversible cerebral vasoconstriction }\end{array}$ & \\
\hline
\end{tabular}

This patient undoubtedly possessed a number of risk factors for developing RCVS. Despite these pre-existing risk factors, the recent use of the antihistamine, loratadine, was the only recognisable change to her history. This link between RCVS and antihistamine use is also supported by another case report [12]. Although it is theoretically plausible, there is limited evidence for the vasoconstrictive effect of antihistamines. Thus, the mechanism for which antihistamines could provoke RCVS is unclear. However, in one study, the vasoconstrictor activity of 4 different 1st-generation antihistamines was demonstrated in the vasculature of rats [13]. Moreover, they found that antihistamines potentiated the effect of catecholamine vasoconstrictor activity [13]. Histamine receptors are found throughout the cerebral vasculature and play a role in regulating vascular tone [14]. Additionally, an association between histamine and other vasoactive neuropeptides has been found. For example, H3 receptors, when stimulated, inhibit the release of intracerebral serotonin $[9,15,16]$. It has also been noted that histamine can inhibit the reuptake of serotonin by brain synapses [17]. Both histamines and serotonin have been shown to play a role in vascular headaches such as migraines through their direct effects on the cerebral vasculature $[9,10,18]$. Migraines are well-known to be caused by vasodilation, and this has been shown to correlate with elevated histamine levels [11]. As a result of this and the success of serotonergic agents in treating migraines, research has gone into studying the use of antihistamine for migraine treatment [14]. As it stands, there is no clear evidence for the role of antihistamines in migraine treatment [9]. However, there is some evidence for their use in combination with other agents [10]. Overall, evidence points towards a complex and possibly indirect relationship between antihistamines and cerebral vascular tone, whereby they may enhance the vasoactive potential of other drugs. In this case, we propose a causal link between antihistamine use and the onset of RCVS, secondary to an accumulation of a number of risk factors. For example, the patient's use of antihistamines may have potentiated the vasoconstrictive nature of other medications

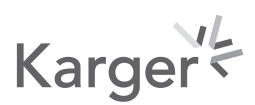


Table 2. Risk factors and potential triggers of RCVS

Changes to hormonal levels

Vasoactive substances

Vascular

Catecholamine secreting tumours

Headache disorders

Environmental

Intracranial disorders or surgery

Miscellaneous

\section{Pregnancy \\ Eclampsia/pre-eclampsia \\ Oral contraceptive pills \\ Hormonal ovarian stimulation}

Illicit drugs: cannabis, ecstasy, amphetamines, cocaine Ergots

Sympathomimetics: diet pills, phenylpropanolamine Serotonergic drugs: serotonin-reuptake inhibitors, triptans

Adrenergic agents - epinephrine, bromocriptine Immunosuppressants: tacrolimus, cyclophosphamide Blood products: blood transfusion, immunoglobulin Others: nicotine, binge drinking, hot chilli peppers

Post-partum angiopathy

Cervical artery dissection

Aortic dissection

Unruptured intracranial aneurysm

Post carotid endartectomy

Cerebral venous thrombosis

Pheochromocytoma

Bronchial carcinoid tumour

Migraines

Primary TCHs

Primary cough headache

Primary headache associated with sexual activity

Exertional headache

High altitude

Cold water exposure

Intracranial hypotension

Intracranial haemorrhage

Spinal subdural haematoma

Neurosurgery

Head trauma

PRES

Hypercalcaemia

Systemic lupus erythematosus

Porphyria

Microangiopathic haemolytic anaemia

Triplet caesarean delivery

Tonsillectomy

Adapted from Refs. [3, 5].

RCVS, reversible cerebral vasoconstriction syndrome; TCH, thunderclap headache; PRES, posterior reversible encephalopathy syndrome.

such as fluoxetine, which in turn led to RCVS. The link between antihistamines and vasoconstriction warrants further research and would be of benefit when it comes to diagnosing the condition through its associated triggers.

Although the true incidence of RCVS is unknown, clinical experience now suggests it may be more common than initially thought, with one study suggesting an incidence of $8.8 \%$ in patients presenting with a TCH [19]. Thus, research into the mechanism and triggers for RCVS 
is essential for accurate and prompt diagnosis. Additionally, increasing clinical awareness of RCVS and its unique characteristics, alongside screening for risk factors, will enable appropriate management of RCVS, reduced complication rates, and hence improved morbidity and mortality.

\section{Statement of Ethics}

The patient was informed of the purpose of the case report and gave her written informed consent for the publication of her case and the publication of her brain images.

\section{Conflict of Interest Statement}

The authors have no conflicts of interest to declare.

\section{Funding Sources}

No funding for this case report was required.

\section{Author Contributions}

The writing of the report was primarily done by Dr. Sara Assadi who drafted, finalized, and edited the report. This was supervised by Dr. Latif Rahman, who initially diagnosed the case of RCVS and proposed the idea of writing a case report. Dr. Mark Kong was solely responsible for the image captions and neuroradiology aspects. Dr. Sukaina Asad (Department of Neurology) supervised and reviewed the paper to add her expertise in neurology.

\section{References}

1 Sheikh HU, Mathew PG. Reversible cerebral vasoconstriction syndrome: updates and new perspectives. Curr Pain Headache Rep. 2014 May;18(5):414.

2 Singhal AB, Hajj-Ali RA, Topcuoglu MA, Fok J, Bena J, Yang D, et al. Reversible cerebral vasoconstriction syndromes: analysis of 139 cases. Arch Neurol. 2011;68(8):1005-12.

3 Chen S-P, Fuh J-L, Wang SJ. Reversible cerebral vasoconstriction syndrome: current and future perspectives. Expert Rev Neurother. 2011;11(9):1265-76.

4 Singhal A, Kasner SE, Rabinstein AA. Reversible cerebral vasoconstriction syndrome. Up to date February 2020. Topic 14075 Version 14.0. 2021.

5 Chen SP, Fuh JL, Wang SJ, Chang FC, Lirng JF, Fang YC, et al. Magnetic resonance angiography in reversible cerebral vasoconstriction syndromes. Ann Neurol. 2010;67(5):648-56.

6 Singhal AB, Caviness VS, Begleiter AF, Mark EJ, Rordorf G, Koroshetz WJ. Cerebral vasoconstriction and stroke after use of serotonergic drugs. Neurology. 2002;58(1):130-3.

7 Rocha EA, Topcuoglu MA, Silva GS, Singhal AB. RCVS2 score and diagnostic approach for reversible cerebral vasoconstriction syndrome. Neurology. 2019 Feb 12;92(7):e639-47. Available from: Epub 2019 Jan 11. PMID: 30635475.

8 Ashina M, Hansen JM, Do TP, Melo-Carrillo A, Burstein R, Moskowitz MA. Migraine and the trigeminovascular system-40 years and counting. Lancet Neurol. 2019;18(8):795-804.

9 Worm J, Falkenberg K, Olesen J. Histamine and migraine revisited: mechanisms and possible drug targets. J Headache Pain. 2019;20:30.

10 Mansfield LE. The role of antihistamine therapy in vascular headaches. J Allergy Clin Immunol. 1990 0ct;86(4 Pt 2):673-6. 
11 Karnushina IL, Palacios JM, Barbin G, Dux E, Joó F, Schwartz JC. Studies on a capillary-rich fraction isolated from brain: histaminic components and characterization of the histamine receptors linked to adenylate cyclase. J Neurochem. 1980;34:1201-8.

12 Guler S. Reversible cerebral vasoconstriction syndrome and recurrent headache triggered by antihistamine use. Agri. 2016;29.

13 Altura BM, Zweifach BW. Antihistamines and vascular reactivity. Am J Physiol. 1965;209:545-9.

14 Saxena PR. The significance of histamine $\mathrm{H} 1$ and $\mathrm{H} 2$ receptors on the carotid vascular bed in the dog. Neurology. $1975 ; 25: 681-7$.

15 Arrang JM, Garbarg M, Schwartz JC. Autoinhibition of brain histamine release mediated by a novel class (H3) of histamine receptor. Nature. 1983;302:832-7.

16 Schlicker E, Betz R, Göthert M. Histamine H3 receptor-mediated inhibition of serotonin release in the rat brain cortex. Naunyn Schmiedebergs Arch Pharmacol. 1988;337:588-90.

17 Young CS, Mason R, Hill SJ. Inhibition by H1-antihistamines of the uptake of noradrenaline and 5-HT into rat brain synaptosomes. Biochem Pharmacol. 1988;37:976-8.

18 Curzon G, Barrie M, Wilkinson MI. Relationships between headache and amine changes after administration of reserpine to migrainous patients. J Neurol Neurosurg Psychiatry. 1969;32:555-61.

19 Grooters GS, Sluzewski M, Tijssen CC. How often is thunderclap headache caused by the reversible cerebral vasoconstriction syndrome? Headache. 2014;54(4):732-5. 